\title{
Laser ablation after stereotactic radiosurgery: a multicenter prospective study in patients with metastatic brain tumors and radiation necrosis
}

\author{
Manmeet Ahluwalia, MD, , Gene H. Barnett, MD, ${ }^{1}$ Di Deng, BS, ${ }^{2}$ Stephen B. Tatter, MD, PhD, ${ }^{3}$ \\ Adrian W. Laxton, MD, ${ }^{3}$ Alireza M. Mohammadi, MD, ${ }^{1}$ Eric Leuthardt, MD, ${ }^{4}$ Roukoz Chamoun, MD, ${ }^{5}$ \\ Kevin Judy, MD, ${ }^{6}$ Anthony Asher, MD, ${ }^{7}$ Marco Essig, MD, PhD, ${ }^{8}$ Jorg Dietrich, MD, PhD, ${ }^{9}$ and \\ Veronica L. Chiang, MD²

\begin{abstract}
'Department of Neurosurgery, Cleveland Clinic, Cleveland, Ohio; '2Department of Neurosurgery, Yale University, New Haven, Connecticut; ${ }^{3}$ Department of Neurosurgery, Wake Forest Medical Center, Winston-Salem, North Carolina; ${ }^{4}$ Department of Neurosurgery, Washington University, St. Louis, Missouri; ${ }^{5}$ Department of Neurosurgery, Kansas University, Kansas City, Kansas; ${ }^{6}$ Department of Neurosurgery, Thomas Jefferson University, Philadelphia, Pennsylvania; ${ }^{7}$ Carolina Neurosurgery and Spine, Charlotte, North Carolina; ${ }^{8}$ Department of Radiology, University of Manitoba, Winnipeg, Manitoba, Canada; and ${ }^{9}$ Department of Neurology, Massachusetts General Hospital, Boston, Massachusetts
\end{abstract}

OBJECTIVE Laser Ablation After Stereotactic Radiosurgery (LAASR) is a multicenter prospective study of laser interstitial thermal (LITT) ablation in patients with radiographic progression after stereotactic radiosurgery for brain metastases. METHODS Patients with a Karnofsky Performance Scale (KPS) score $\geq 60$, an age $>18$ years, and surgical eligibility were included in this study. The primary outcome was local progression-free survival (PFS) assessed using the Response Assessment in Neuro-Oncology Brain Metastases (RANO-BM) criteria. Secondary outcomes were overall survival (OS), procedure safety, neurocognitive function, and quality of life.

RESULTS Forty-two patients-19 with biopsy-proven radiation necrosis, 20 with recurrent tumor, and 3 with no diagnosis-were enrolled. The median age was 60 years, $64 \%$ of the subjects were female, and the median baseline KPS score was 85 . Mean lesion volume was $6.4 \mathrm{~cm}^{3}$ (range $0.4-38.6 \mathrm{~cm}^{3}$ ). There was no significant difference in length of stay between the recurrent tumor and radiation necrosis patients (median 2.3 vs 1.7 days, respectively). Progression-free survival and OS rates were $74 \%$ (20/27) and $72 \%$, respectively, at 26 weeks. Thirty percent of subjects were able to stop or reduce steroid usage by 12 weeks after surgery. Median KPS score, quality of life, and neurocognitive results did not change significantly for either group over the duration of survival. Adverse events were also similar for the two groups, with no significant difference in the overall event rate. There was a 12-week PFS and OS advantage for the radiation necrosis patients compared with the recurrent tumor or tumor progression patients.

CONCLUSIONS In this study, in which enrolled patients had few alternative options for salvage treatment, LITT ablation stabilized the KPS score, preserved quality of life and cognition, had a steroid-sparing effect, and was performed safely in the majority of cases.

Clinical trial registration no.: NCT01651078 (clinicaltrials.gov)

https://thejns.org/doi/abs/10.3171/2017.11.JNS171273

KEYWORDS laser interstitial thermal therapy; NeuroBlate; metastatic brain tumor; radiation necrosis; Laser Ablation After Stereotactic Radiosurgery; LAASR; oncology

ABBREVIATIONS CNS = central nervous system; CR = complete response; $\mathrm{FACT}-\mathrm{Br}=$ Functional Assessment of Cancer Therapy-Brain; HVLT-R = Hopkins Verbal Learning Test-Revised; KPS = Karnofksy Performance Scale; LAASR = Laser Ablation After Stereotactic Radiosurgery; LITT = laser interstitial thermal therapy; MMSE = Mini-Mental State Examination; $O S=$ overall survival; $P D=$ progressive disease; $P F S=$ progression-free survival; $P R=$ partial response; $Q O L=$ quality of life; $R A N O-B M=$ Response Assessment in Neuro-Oncology Brain Metastases; RN = radiation necrosis; SRS = stereotactic radiosurgery.

SUBMITTED May 29, 2017. ACCEPTED November 4, 2017.

INCLUDE WHEN CITING Published online May 4, 2018; DOI: 10.3171/2017.11.JNS171273. 
$\mathrm{S}$ TEREOTACTIC radiosurgery (SRS) is the standard first-line treatment for most patients with a limited number of brain metastases. ${ }^{7}$ However, a portion of lesions treated with SRS will subsequently regrow, which can lead to neurological deterioration. ${ }^{17}$ This regrowth, seen on radiological follow-up, can be attributable to either true tumor recurrence/progression or an inflammatory nonneoplastic adverse effect of prior radiosurgery known as radiation necrosis (RN). Patients can be symptomatic from lesions in either scenario. Sneed et al. reported a 1-year local recurrence rate of $9.2 \%$ and a 1-year cumulative incidence rate of adverse radiation effects of up to $14 \% .{ }^{17}$ Other studies have reported various rates of local recurrence ranging from $7 \%$ to $27.5 \%$ and rates of symptomatic radionecrosis ranging from $2 \%$ to $10 \% .^{18}$ Clinical outcome for patients with progressive brain metastasis or $\mathrm{RN}$ is generally poor and thought to be associated with a shortened lifespan and reduction in quality of life (QOL) ${ }^{18}$

In patients with tumor regrowth, chemotherapy may improve survival but can significantly impact a patient's QOL and should have no beneficial effect on RN. Medical treatments for $\mathrm{RN}$ can be expensive or have poorly tolerated adverse effects. Resection of both pathological entities has been shown to be beneficial in local control of the regrowing lesion if it is amenable to traditional craniotomy, but recovery from the craniotomy itself can take 4-8 weeks, which can be undesirable in a patient population with a limited lifespan..$^{10}$ Further, patients undergoing repeat craniotomies are more likely to experience depression (20\%) or systemic infection (4\%) and to have a worse immediate postoperative neurological status $(18 \%){ }^{5}$ In addition, repeat irradiation of lesions failing prior radiosurgery is associated with higher rates of RN. ${ }^{17}$

Anecdotally, the option of MRI-guided laser interstitial thermal therapy (LITT) has been effective for the treatment of both pathological entities after SRS when craniotomy is not feasible because of either lesion location or concerns regarding medical comorbidities. . $^{1,3,6,11,12,14,19,20}$ Results from a phase I clinical trial using the NeuroBlate system (Monteris Medical Inc.) showed that the device provides neurosurgeons with a minimally invasive surgical option for tumor ablation. ${ }^{15}$ An earlier study in 16 glioblastoma patients suggested that LITT offers a survival advantage over medical management or chemotherapy alone. ${ }^{13}$

Thus, the Laser Ablation After Stereotactic Radiosurgery (LAASR) study was designed to prospectively determine the safety, efficacy, and outcome of laser ablation for the treatment of recurrent brain metastases or RN following prior SRS.

\section{Methods \\ Patients}

This multisite, open-label phase II study of the FDAcleared NeuroBlate system was performed across 6 centers in the United States with prior experience using laser ablation in the brain: 1) Cleveland Clinic, 2) Wake Forest University, 3) University of Kansas, 4) Washington University, 5) Thomas Jefferson University, and 6) Yale University. The study was registered with the ClinicalTrials. gov database (http://clinicaltrials.gov), and its registration no. is NCT01651078. Institutional review board approval was obtained at each site, and consent for the study was obtained per each site's IRB requirements. Brain metastases patients with evidence of radiographic lesion growth following prior treatment with SRS were recruited into the study if they had a Karnofsky Performance Scale (KPS) score $\geq 60$, an age $>18$ years, and metastatic disease from a known primary cancer type and were good surgical candidates. Patients were excluded for any of the following reasons: 1) pregnancy; 2) leptomeningeal disease; 3) progressive symptoms due to mass effect despite steroids; 4) uncontrolled hypertension or cardiac dysrhythmia; 5) intracranial hemorrhage within the past 6 weeks; 6) serious concurrent systemic medical conditions; 7) concerns about coagulopathy including bone marrow suppression, thrombocytopenia, and the need for ongoing anticoagulation or antiplatelet therapy; and 8) an inability to undergo MRI because the scanner could not accommodate the patient's physical dimensions or other reasons why MRI could not be performed. Nontarget lesions were allowed to be present on enrollment MRI as long as they were not expected (in the investigator's judgment) to contribute to symptoms during the course of the study or to confound outcome interpretation.

Radiographic regrowth was defined by standard reports from a clinical radiologist at each institution. The decision to treat the radiological lesion with LITT was determined by a tumor board at each site. Factors used to decide to proceed with LITT included 1) the need for a diagnosis, 2) progression of symptoms (despite steroids) clearly attributable to a single lesion that would probably be ameliorated by resection, and 3) continued lesion growth over serial brain MRI sequences with a lesion size that was believed to be amenable to LITT based on individual center experience. Patient demographics, clinical cancer data, and all medical, radiation, and surgical treatment data were collected both prior and subsequent to LITT. The status of extracranial disease was defined by body imaging as reported at each institution. In addition, neurological functional data before and after LITT were collected.

\section{Surgical Management}

Preoperative gadolinium contrast-enhanced T1-weighted volumetric images were used for visualization, planning, and management. A biopsy was obtained in all cases to determine if lesion growth was attributable to tumor recurrence or RN. Pathology results of the biopsies were obtained from the pathology reports at each institution. All 6 centers used the NeuroBlate system for LITT, as previously described..$^{15}$ The system employs a robotically controlled 1064-nm laser probe and uses MRI thermometry to inform the surgeon of predicted zones of protein denaturation and cell death.

\section{Outcome Measures}

The primary outcome was local central nervous system (CNS) progression-free survival (PFS) as defined by standard Response Assessment in Neuro-Oncology Brain Metastases (RANO-BM) criteria. ${ }^{9}$ All pre- and postoperative images were independently reviewed by two neuroradiolo- 
gists at an MRI core laboratory (University of Manitoba). Postoperative imaging was also used to determine if target lesions were totally or subtotally treated. Secondary outcomes were overall survival (OS), functional outcome as measured by change in the KPS score, neurocognition as measured by the Hopkins Verbal Learning Test-Revised (HVLT-R) and Mini-Mental State Examination (MMSE), QOL as measured by the Functional Assessment of Cancer Therapy-Brain (FACT-Br), and rate of procedure-related complications. The HVLT-R, MMSE, and FACT-Br data along with neurological outcomes were collected at 3 and 6 months (12 and 26 weeks) post-LITT.

\section{Statistical Analysis}

Statistical analysis was performed using SAS software, version 9.1 (SAS Institute Inc.). The $\mathrm{p}$ values for discrete outcomes represent the probability of differences in the probability of an event over time using ANOVA and are presented as frequencies. The $p$ values for continuous outcomes represent the probability of differences in mean outcomes over time using ANOVA and are presented as the median and interquartile range. The Kaplan-Meier method was used to obtain an estimate of OS. A multivariate analysis for discharge time was performed using logarithmic regression, and the following variables were included: confirmed biopsy, smoking status, heart disease, steroid use at baseline, and tumor volume. A p value < 0.05 was considered to indicate statistical significance.

\section{Results \\ Overall Demographics and Procedure}

Forty-four patients were screened into the LAASR study between October 2012 and December 2015, but only 42 patients were treated with LITT because 2 patients did not fulfill study entry criteria. Mean age of the patients was 58.5 years (range 32-74 years), and 27 (64.3\%) of the 42 patients were female. The primary tumor pathology was non-small cell lung cancer in $43 \%$ of patients, breast cancer in $17 \%$, melanoma in $10 \%$, and other tumor types in 30\% (Table 1). Median baseline KPS score was 85 (range $60-100)$, and $43 \%$ of patients $(18 / 42)$ were on steroids at study entry. Twelve patients $(28.6 \%)$ had previously undergone craniotomy for tumor resection, and 7 patients $(16.7 \%)$ had received previous whole-brain radiation therapy. Only 1 patient was receiving ongoing chemotherapy prior to LITT.

Mean lesion volume was $6.4 \mathrm{~cm}^{3}$ (range $0.4-38.6 \mathrm{~cm}^{3}$ ). Tumor was in the frontal lobe in $41 \%$ of cases, parietal lobe in $29 \%$, cerebellum in $14 \%$, and other in $16 \%$ (occipital lobe, temporal lobe, thalamus, and other deep nuclei). Pathological analysis of the biopsy at the time of LITT showed recurrent tumor in 20 cases (48\%), RN without evidence of tumor in 19 cases (45\%), and was nondiagnostic in 3 cases (7\%). Median procedure time was 3 hours (range 1.4-9.7 hours), and median length of hospital stay was 2 days (range $0.4-12$ days).

After LITT, of the 20 patients with a biopsy diagnosis of recurrent tumor, postoperative chemotherapy/medical therapy information was available for only 5 , who received ongoing treatment with ipilimumab + nivolumab
(1), dabrafenib (1), trastuzumab (1), pemetrexed (1), and lapatinib (1).

In comparing patients with recurrent brain metastases to those with RN, we found no statistically significant differences in age, sex, race, medical comorbidities, primary cancer type, baseline KPS score, percentage using steroids at baseline, prior local treatment, tumor location, or tumor volume (Table 1). Median length of stay was 2.3 days (range 1-12 days) for tumor patients and 1.7 days (0.5-6.5 days) for $\mathrm{RN}$ patients $(\mathrm{p}=0.11)$.

\section{Outcome}

Twenty-seven patients (64\%) completed the 12-week follow-up, and 16 patients (38\%) completed the full 26week follow-up (Fig. 1). Therefore, imaging, cognition, and QOL outcomes as well as complication data were only available for some of the patients (Table 2), although OS data are available for all patients.

\section{Local PFS}

Local PFS for the group with available data was $74 \%$ (20/27) at 12 weeks and through the last follow-up (12-26 weeks). At 12 weeks, $15 \%$ (4/27) of the LITT-treated lesions were stable, $22 \%(6 / 27)$ had a partial response (PR), and $37 \%(10 / 27)$ had a complete response (CR). Ultimately, 48\% (13/27) of LITT-treated lesions for which followup was available showed a CR. At both the 12- and 26week time points, $26 \%$ of the lesions continued to progress despite LITT treatment (Table 2).

Additional analysis of local PFS at 12 weeks was performed based on whether the lesion was totally or subtotally ablated. Ablation data were only available for 9 RN lesions and 12 tumor regrowth lesions. Of the lesions that had received total ablation, $100 \%$ (4/4) of the RN lesions and $75 \%(3 / 4)$ of the tumor lesions showed a CR (Table 2). The remaining $25 \%(1 / 4)$ of tumors that were totally ablated showed a PR. These findings contrast with those for the tumors that had received subtotal ablation: 0\% showed a CR and 63\% (5/8) showed progressive disease (PD). In total, $7(87.5 \%)$ of 8 lesions that were totally ablated demonstrated radiographic CR compared with $0(0 \%)$ of 13 lesions that were subtotally ablated. Achieving a radiographic CR was shown to be statistically significantly related to having received total ablation $(\mathrm{p}<0.001)$. In addition, of the lesions demonstrating PD at 12 weeks, all had been confirmed as tumor tissue on biopsy and had undergone incomplete ablation at the time of LITT treatment.

Comparing the two groups based on pathology, we found local PFS was statistically different at 12 weeks $(100 \%$ for RN vs $54 \%$ for tumor, $p=0.016)$ but not at the last follow-up beyond 12 weeks ( $91 \%$ for RN vs $62 \%$ for tumor, $p=0.166$; Table 3). The local PFS increase in the tumor group at the last follow-up compared with 12 weeks was attributable to a single tumor case that was categorized as PD at 12 weeks according to the RANO$\mathrm{BM}$ criteria but subsequently became a CR at the 26-week evaluation after radiation treatment for progression. This patient also received trastuzumab during follow-up. The tumor patient recorded as receiving the ipilimumab + nivolumab combination had a radiographic $\mathrm{CR}$ at both the 12- and 26-week follow-ups. 
TABLE 1. Baseline patient demographics and disease characteristics

\begin{tabular}{|c|c|c|c|c|}
\hline Parameter & Regrowing Tumor Group & Radiation Necrosis Group & p Value* & All Patients \\
\hline No. of patients & 20 & 19 & & 42 \\
\hline \multicolumn{5}{|l|}{ Sex } \\
\hline Female & $70.0 \%$ & $63.2 \%$ & \multirow[t]{2}{*}{0.7411} & $64.3 \%$ \\
\hline Male & $30.0 \%$ & $36.8 \%$ & & $35.7 \%$ \\
\hline Age in yrs & & & \multicolumn{2}{|l|}{0.9980} \\
\hline Mean & $58.9 \pm 11.2$ & $58.8 \pm 7.5$ & & $58.5 \pm 9.9$ \\
\hline Median (range) & $60.0(32.0-74.0)$ & $58.0(49.0-72.0)$ & & $60.0(32.0-74.0)$ \\
\hline \multicolumn{5}{|l|}{ Race } \\
\hline White† & $85.0 \%$ & $89.5 \%$ & \multirow[t]{3}{*}{0.6050} & $88.1 \%$ \\
\hline Asian & $0 \%$ & $5.3 \%$ & & $2.4 \%$ \\
\hline African American & $15.0 \%$ & $5.3 \%$ & & $9.5 \%$ \\
\hline \multicolumn{5}{|l|}{ Smoking status } \\
\hline Current & $30.0 \%$ & $10.5 \%$ & \multirow[t]{3}{*}{0.3466} & $19.0 \%$ \\
\hline Never & $25.0 \%$ & $36.8 \%$ & & $31.0 \%$ \\
\hline Quit >6 mos ago & $45.0 \%$ & $52.6 \%$ & & $50.0 \%$ \\
\hline \multicolumn{5}{|l|}{ History of heart disease } \\
\hline Current & $30.0 \%$ & $26.3 \%$ & \multirow[t]{3}{*}{1.0000} & $26.2 \%$ \\
\hline Never & $65.0 \%$ & $73.7 \%$ & & $69.0 \%$ \\
\hline Prior & $5.0 \%$ & $0 \%$ & & $4.8 \%$ \\
\hline \multicolumn{5}{|l|}{ Primary tumor site } \\
\hline Breast & $10.0 \%$ & $26.3 \%$ & \multirow[t]{7}{*}{0.6883} & $16.7 \%$ \\
\hline GYN & $0 \%$ & $0 \%$ & & $2.4 \%$ \\
\hline NSCLC & $50.0 \%$ & $36.8 \%$ & & $42.9 \%$ \\
\hline SCLC & $5.0 \%$ & $5.3 \%$ & & $4.8 \%$ \\
\hline Melanoma & $10.0 \%$ & $10.5 \%$ & & $9.5 \%$ \\
\hline Other & $25.0 \%$ & $15.8 \%$ & & $21.4 \%$ \\
\hline Renal & $0 \%$ & $5.3 \%$ & & $2.4 \%$ \\
\hline Baseline KPS score & & & \multicolumn{2}{|l|}{0.1986} \\
\hline Mean & $79.5 \pm 11.9$ & $84.7 \pm 13.1$ & & $82.1 \pm 13.0$ \\
\hline Median (range) & $80.0(60.0-100.0)$ & $90.0(60.0-100.0)$ & & $85.0(60.0-100.0)$ \\
\hline Steroid use at baseline & $40.0 \%$ & $36.8 \%$ & 1.0000 & $42.9 \%$ \\
\hline No. of lesions & 23 & 19 & & 45 \\
\hline Mean lesion vol in $\mathrm{cm}^{3}$ (range) & $7.1 \pm 8.7(0.8-38.6)$ & $5.5 \pm 3.9(0.4-13.2)$ & 0.4185 & $6.4 \pm 6.7(0.4-38.6)$ \\
\hline
\end{tabular}

GYN = gynecological; NSCLC = non-small cell lung cancer; $\mathrm{SCLC}=$ small cell lung cancer.

Data expressed as mean \pm standard deviation, median (range), or $\%$.

* The value calculated from the 2-sample t-test for continuous summary and Fisher's exact test for categorical summary.

$\dagger 100 \%$ non-Hispanic ethnicity.

Only 8/42 (19\%) patients experienced local lesional progression during the course of the study, and the majority of these patients had biopsy-confirmed metastatic tumor. Patients who went on to have progressive lesions did have a lower baseline KPS score than those without progressive lesions (median 70 vs 90, $p=0.0037$ ); however, there were no other significant differences in baseline characteristics. Patients who did have radiographic local lesion progression were more likely to be moved to hospice or to die during the course of the study (HR 0.12, p = 0.027) after the 12-week follow-up.

\section{Overall Survival and Neurological Outcome}

Overall survival for the whole group was $86.5 \%$ at 12 weeks and $72.2 \%$ at 26 weeks. For tumor patients, OS was $71 \%$ at 12 weeks and $64.5 \%$ at 26 weeks. For RN patients, OS survival was $100 \%$ at 12 weeks and $82.1 \%$ at 26 weeks. Overall survival was significantly different between the two groups at 12 weeks $(\mathrm{p}=0.02)$ but not at 26 weeks $(\mathrm{p}=$ 0.09; Fig. 2).

The median change in the KPS score was 0 (range -40 to +20$)$, and $60 \%(25 / 42)$ of the patients had stable or improved KPS at their last follow-up. Twenty-seven percent of the patients (3/11) had recorded neurological improvement from the baseline exam to the last visit, and $31 \%$ were able to stop or reduce steroid usage by the 12 -week follow-up. No statistically significant difference in these percentages for either neurological change or change in 


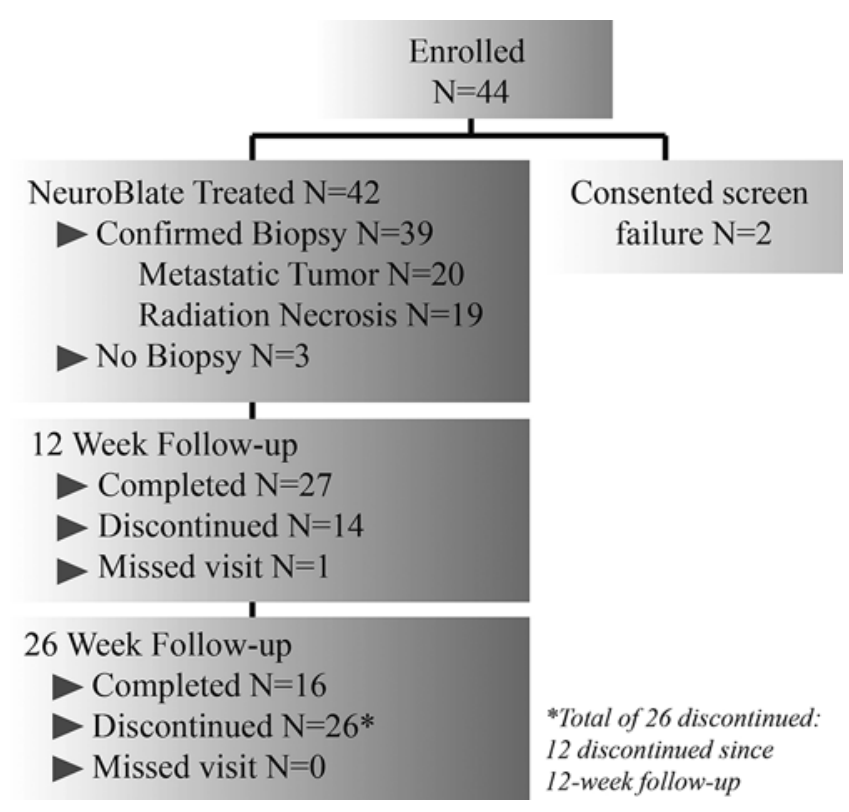

FIG. 1. Trial flow chart.

steroid use after LITT were seen between the tumor and RN groups. Figure 3 shows FLAIR and gadolinium MRI examples taken pre-LITT and at 26 weeks after LITT.

\section{Cognition and QOL}

No significant change was noted in the HVLT-R or MMSE scores between baseline and the 12- or 26-week results for the overall group. In addition, no significant change was noted in the overall FACT-Br scores at either time point; however, when the scale was divided into its subcategories, a statistically significant decline in Social Well-Being (SWB) scores was seen at 12 weeks (median -1.0 , range -12 to $8.2, p=0.0443$ ) and in Emotional WellBeing (EWB) scores at 26 weeks (median 3.5, range -5 to $11, \mathrm{p}=0.0170)$. This decline in EWB and SWB scores was seen in both tumor and RN patients, and the lack of a correlation between disease progression and worsened
TABLE 3. Progression-free survival by pathological analysis of biopsy specimen

\begin{tabular}{lcccc}
\hline \multicolumn{1}{c}{ Parameter } & $\begin{array}{c}\text { All } \\
\text { Subjects }\end{array}$ & $\begin{array}{c}\text { Regrowing } \\
\text { Tumor Group }\end{array}$ & $\begin{array}{c}\text { Radiation } \\
\text { Necrosis Group }\end{array}$ & $\begin{array}{c}p \\
\text { Value }\end{array}$ \\
\hline No. of patients & 24 & 13 & 11 & \\
\hline 12 wks & $75 \%(18 / 24)$ & $54 \%(7 / 13)$ & $100 \%(11 / 11)$ & 0.0162 \\
\hline Last FU & $75 \%(18 / 24)$ & $62 \%(8 / 13)$ & $90.9 \%(10 / 11)$ & 0.1660 \\
\hline * Three of 27 patients with 12-week follow-up data had nondiagnostic biopsy \\
and were excluded from this table.
\end{tabular}

QOL was confirmed using interaction plots for correlations. However, increased FACT-Br scores (indicative of worsening QOL) were significantly associated with a greater likelihood of death or being moved to hospice (HR $=0.972, p=0.044)$. In addition, there was no significant effect of prior whole-brain radiation therapy on cognition or QOL.

\section{Complications}

Overall, 35 of 42 patients developed adverse events during the study. Adverse events were defined as any undesirable medical occurrence in a clinical trial patient, regardless of whether it was related to the device or not, that included a clinical sign, symptom, or condition and/or an observed unintended technical performance or performance outcome of the device. No unanticipated adverse device events occurred during the study. Five patients (12\%) incurred an immediate LITT-related neurological complication (Table 4). In 4 of these cases, LITT had been performed adjacent to motor, sensory, and/or speech areas, and postoperatively these patients had new or worsened neurological deficits. In the fifth case, an intracerebral hemorrhage occurred but did not result in the need for craniotomy or a new neurological deficit.

During the subsequent postoperative admission, 14 patients $(33 \%)$ had surgery-related adverse events. The most common adverse events were transient neurological symptoms and seizures (7), nausea/vomiting (3), cardiopulmonary events (3), and an asymptomatic increase in radio-

TABLE 2. RANO-BM outcomes by time point and ablation coverage

\begin{tabular}{|c|c|c|c|c|}
\hline Outcome & Complete Response & Partial Response & Stable Disease & Progressive Disease \\
\hline \multicolumn{5}{|l|}{ PFS overall cohort } \\
\hline 12-wk local lesion response $(n=27)$ & $37 \%(10 / 27)$ & $22 \%(6 / 27)$ & $15 \%(4 / 27)$ & $26 \%(7 / 27)$ \\
\hline 12-wk response & & $74 \%$ PFS & & $26 \%$ progression \\
\hline Last FU* local lesion response $(n=27)$ & $48 \%(13 / 27)$ & $15 \%(4 / 27)$ & $11 \%(3 / 27)$ & $26 \%(7 / 27)$ \\
\hline Last FU* response & & $74 \%$ PFS & & $26 \%$ progression \\
\hline \multicolumn{5}{|c|}{ 12-wk local lesion progression by pathology \& ablative coverage } \\
\hline RN, total ablation $(n=4)$ & $100 \%(4 / 4)$ & 0 & 0 & 0 \\
\hline RN, subtotal ablation $(n=5)$ & 0 & $60 \%(3 / 5)$ & $40 \%(2 / 5)$ & 0 \\
\hline Tumor, total ablation $(n=4)$ & $75 \%(3 / 4)$ & $25 \%(1 / 4)$ & 0 & 0 \\
\hline Tumor, subtotal ablation $(\mathrm{n}=8)$ & 0 & $12.5 \%(1 / 8)$ & $25 \%(2 / 8)$ & $62.5 \%(5 / 8)$ \\
\hline
\end{tabular}

$\mathrm{FU}=$ follow-up; $\mathrm{n}$ = number of patients.

* Anytime from 12 to 26 weeks: 16/42 (38\%) patients submitted follow-up imaging at 26 weeks, and 27 total patients submitted imaging for analysis. 


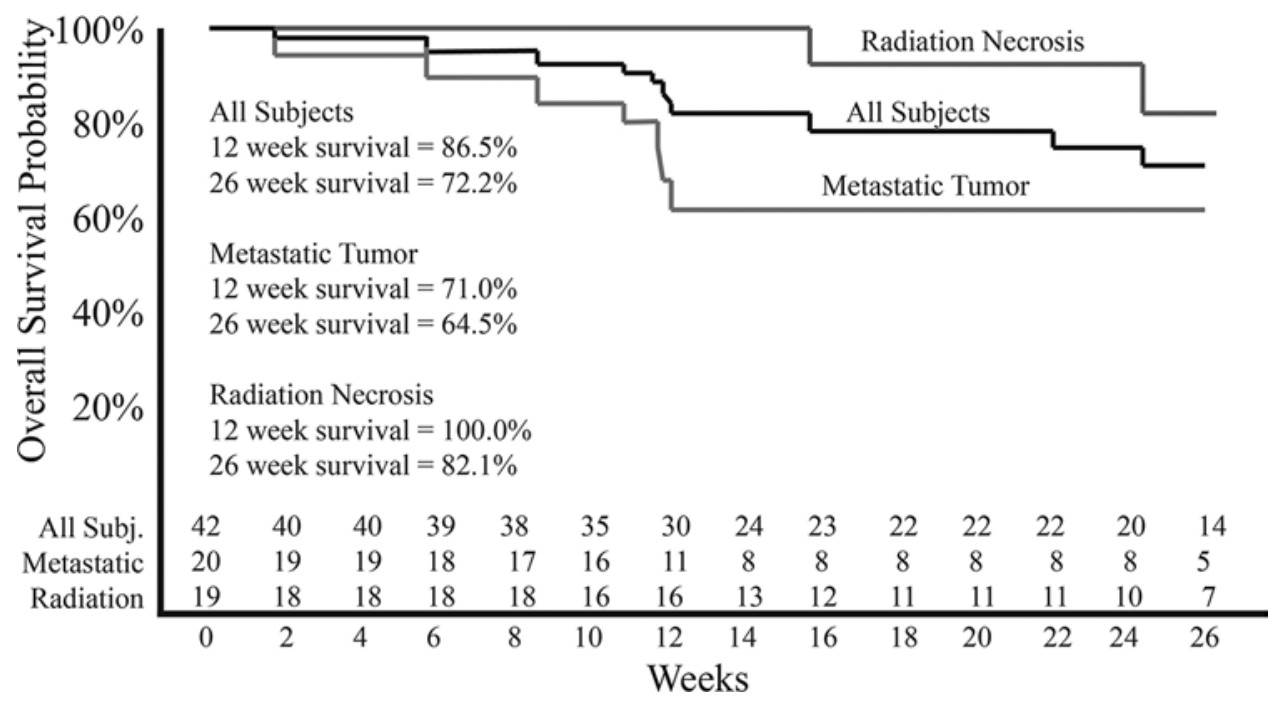

FIG. 2. Kaplan-Meier survival curves. Subj. = subjects.

graphically defined cerebral edema (3). Twenty-two of 42 patients had adverse events recorded beyond 1 month after surgery-due to progression of disease in 10-and only 1 patient was readmitted within 90 days for pulmonary embolism.

There was no significant difference in the rate of overall adverse events between the tumor group and $\mathrm{RN}$ group ( $\mathrm{p}$ $=0.69$ ).

\section{Discussion}

Patients who develop radiographic lesion recurrence or progression after SRS treatment for brain metastasis can benefit from surgical management, especially if the cause of the radiographic regrowth is RN. ${ }^{10}$ In patients who cannot or are unwilling to undergo craniotomy, LITT can be performed as an alternative to craniotomy and has been anecdotally reported to be efficacious in managing this problem.

The first report of LITT use in this patient population appeared in 2008, in which Carpentier et al. described the feasibility and safety of LITT treatment for regrowing metastases in 4 patients. $^{3}$ While only 3 of the 4 patients had
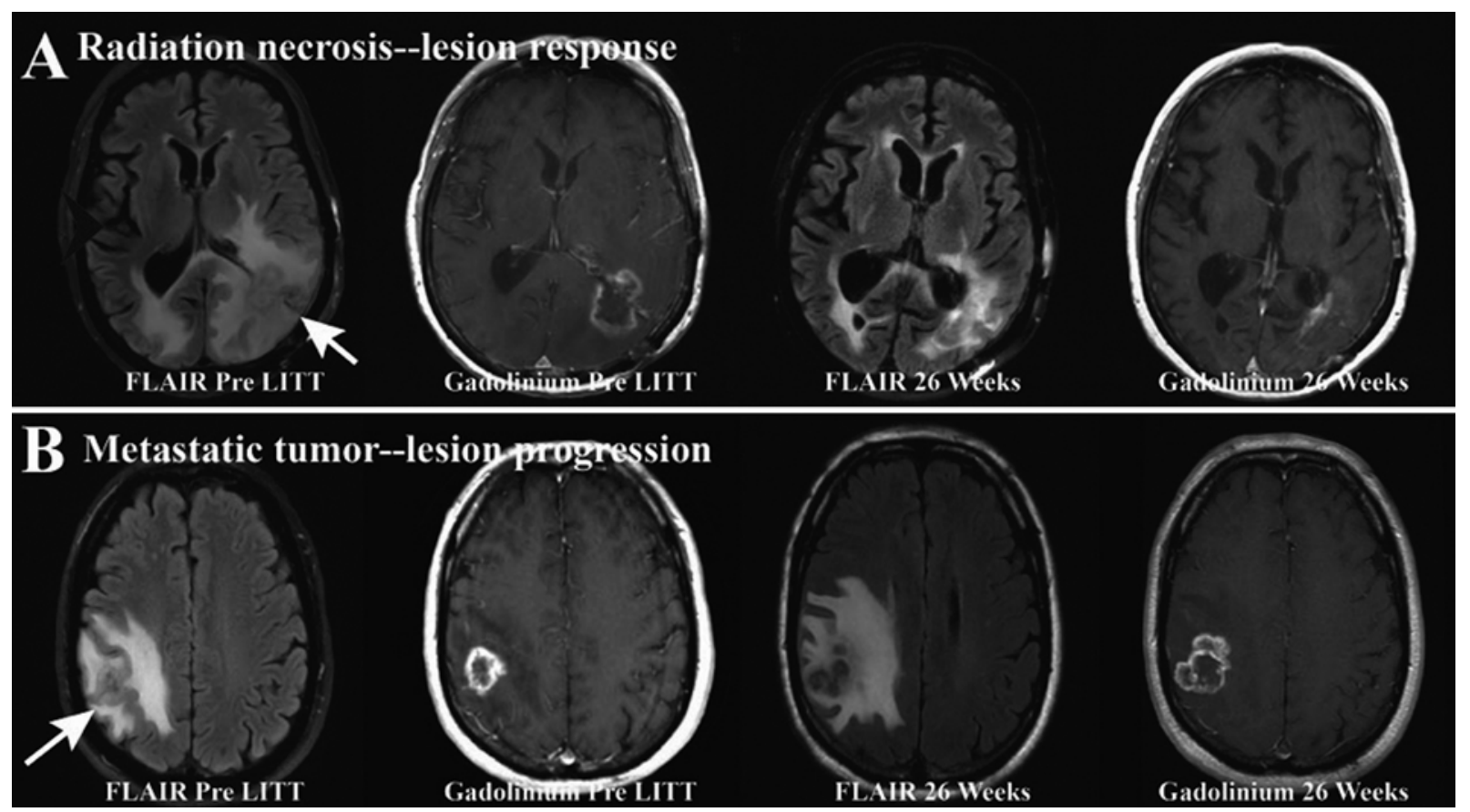

FIG. 3. Axial FLAIR and gadolinium MRI examples of RN demonstrating a response to LITT (A) and metastatic tumor with lesion progression after LITT (B). Arrows indicate lesions in the FLAIR pre-LITT images. 
TABLE 4. Adverse events associated with LITT

\begin{tabular}{lccc}
\hline \multicolumn{1}{c}{ Adverse Events } & $\begin{array}{c}\text { Regrowing } \\
\text { Tumor } \\
\text { Group }\end{array}$ & $\begin{array}{c}\text { Radiation } \\
\text { Necrosis } \\
\text { Group }\end{array}$ & $\begin{array}{c}\text { Overall } \\
\text { Patients }\end{array}$ \\
\hline No. of patients & 20 & 19 & 42 \\
\hline \% total patients w/ events & 15.0 & 10.5 & 11.9 \\
\hline Complete hemiparesis (\%) & 0 & 5.3 & 2.4 \\
\hline Headache (\%) & 0 & 5.3 & 2.4 \\
\hline $\begin{array}{l}\text { Hemineglect and It-sided } \\
\text { weakness (\%) }\end{array}$ & 0 & 5.3 & 2.4 \\
\hline Immediate postop effects (\%)* & 5.0 & 0 & 2.4 \\
\hline Intracerebral hemorrhage (\%) & 5.0 & 0 & 2.4 \\
\hline Weakness (\%) & 5.0 & 0 & 2.4 \\
\hline
\end{tabular}

Adverse event relatedness was based on event adjudication. Adverse events are site reported.

* Immediately postoperatively, the patient presented with left upper-extremity weakness, slight left facial droop, full body itchiness, and persistent dyspraxia (outcome: recovering/resolving).

complete lesional treatment, all had improvement in their KPS score 3 months after surgery. Since then, several other investigators have detailed their experience (single cases or case series) and have described variable success with LITT for the treatment of both biopsy-proven regrowing tumor ${ }^{8,19,20}$ and biopsy-proven RN. ${ }^{4,11,16}$

Therefore, the current study was performed to prospectively determine the safety, efficacy, and outcome of LITT for regrowing SRS-treated lesions. Our results confirm that LITT can be effective in controlling both recurrent/progressive brain metastases and RN following SRS. Overall survival for the whole group was $86.5 \%$ at 12 weeks and $72.2 \%$ at 26 weeks. Survival at 12 weeks was significantly better in the patients with biopsy-proven RN than in those whose biopsy showed recurrent tumor, although there was no statistical significance by 26 weeks after LITT-probably due to the sharp fall-off in patients remaining in the study beyond 12 weeks.

In cases in which LITT was successful in this study, radiographic control or resolution of the concerning lesion was demonstrated in all but 1 case by 12 weeks. Radiographic CR was more likely to occur if total ablation of the lesion had been achieved ( $p<0.001)$, and local PFS was significantly better for biopsy-proven $\mathrm{RN}$ lesions (100\% at 12 weeks) than for biopsy-proven tumor $(54 \%$ at 12 weeks; $p=0.016$ ). While a median PFS duration of 37 weeks was previously reported by Rao et al., ${ }^{12}$ these authors had not performed biopsies; therefore, the differential proportion of progressive brain metastases or RN in that study remains unknown. Smith et al ${ }^{16}$ reported a median PFS of 11.4 months in a group of patients treated with LITT for biopsy-proven RN, a result not inconsistent with the findings in our study. In addition, Ali et al..$^{1}$ showed that in patients with biopsy-proven recurrent tumor, postLITT treatment with hypofractionated radiation resulted in $100 \%$ lesion control compared with only $57 \%$ control if additional radiation was not administered following LITT. Together, the data suggest that it is important to biopsy lesions showing radiological evidence of growth at the time of LITT to guide the discussion of the need for additional post-LITT treatment.

This study also demonstrated that successful LITT can result in stabilization of the KPS score, preservation of cognition and QOL, and reduction in the use of steroids. While there is limited literature with which to compare our findings, one can interpret our results as an improvement in outcome compared with the expected progressive decline in function of this patient population due to continued lesion growth.

Neurological complications related to LITT were seen in $12 \%$ of patients. It is known that LITT adjacent to the subcortical motor sensory fibers can cause permanent neurological deficits. A recent publication by Sharma et al. ${ }^{14}$ showed that overlap between the area of LITT and the corticospinal tracts, as defined by diffusion tensor imaging fiber tracking, can result in a permanent deficit. Patients with lesions close to the corticospinal tracts may benefit from functional imaging studies (for example, tractography) prior to LITT to guide intraoperative treatment.

This study, while prospective, is limited by its small sample size, lack of a matched cohort treated using either craniotomy or best medical therapy, and high study dropout rate after the completion of LITT treatment. Despite the fact that all the study centers have robust radiosurgery programs and anecdotally report a significant number of patients with regrowing SRS-treated lesions, it was surprisingly difficult to accrue to and then maintain the patient population in this trial. While the survival data are robust, the effects of LITT on cognition, QOL, steroid use, and functional status remain inconclusive due to possible bias created by the significant loss of data over time. In addition, it is unclear if the study population is fully reflective of the LITT-eligible population at large as only 1 patient was receiving systemic chemotherapy at the time of radiographic lesion regrowth. This lack of systemic treatment could reflect a very salvageable population with minimal systemic disease, but the results may then be less applicable to patients receiving ongoing chemotherapy.

\section{Conclusions}

In summary, this prospective study confirmed that LITT is a low-risk surgical procedure that can control radiographic lesion (either tumor or RN) growth after SRS in patients with brain metastases and should be considered in those who are surgically eligible. Additional benefits may include minimizing cognitive decline, stabilizing QOL and functional status, and allowing the cessation of steroids in some patients. Further studies with a control group for better characterization of possible benefits are warranted. Biopsy at the time of LITT is recommended to guide follow-up care decisions. For patients with RN, LITT is a good treatment option. Regardless of whether a lesion was totally or subtotally ablated, LITT resulted in close to $100 \%$ lesion control and $>80 \%$ survival at 6 months. In patients with recurrent tumor, however, while LITT can achieve local control if the lesion is totally ablated, the rate of local control in our study was far lower than for RN; therefore, additional therapeutic intervention such as further local radiation or systemic therapies should be 
considered postoperatively. Larger studies with longer follow-up and comparison with the natural history of lesions in untreated patients are needed to elucidate which factors may best predict improved outcomes after LITT and the timing of consolidative therapy. The difficulty with which patients may accrue to such a study, however, may need to be factored into the design of future studies.

\section{Acknowledgments}

We thank Nissa Mollema, PhD, of Monteris Medical for her careful review of this manuscript and editorial assistance.

\section{References}

1. Ali MA, Carroll KT, Rennert RC, Hamelin T, Chang L, Lemkuil BP, et al: Stereotactic laser ablation as treatment for brain metastases that recur after stereotactic radiosurgery: a multiinstitutional experience. Neurosurg Focus 41(4):E11, 2016

2. Aoyama H, Shirato H, Tago M, Nakagawa K, Toyoda T, Hatano K, et al: Stereotactic radiosurgery plus whole-brain radiation therapy vs stereotactic radiosurgery alone for treatment of brain metastases: a randomized controlled trial. JAMA 295:2483-2491, 2006

3. Carpentier A, McNichols RJ, Stafford RJ, Itzcovitz J, Guichard JP, Reizine D, et al: Real-time magnetic resonance-guided laser thermal therapy for focal metastatic brain tumors. Neurosurgery 63 (1 Suppl 1):ONS21-ONS29, 2008

4. Chan AY, Tran DK, Gill AS, Hsu FP, Vadera S: Stereotactic robot-assisted MRI-guided laser thermal ablation of radiation necrosis in the posterior cranial fossa: technical note. Neurosurg Focus 41(4):E5, 2016

5. Chang SM, Parney IF, McDermott M, Barker FG II, Schmidt $\mathrm{MH}$, Huang W, et al: Perioperative complications and neurological outcomes of first and second craniotomies among patients enrolled in the Glioma Outcome Project. J Neurosurg 98:1175-1181, 2003

6. Fabiano AJ, Qiu J: Delayed failure of laser-induced interstitial thermotherapy for postradiosurgery brain metastases. World Neurosurg 82:e559-e563, 2014

7. Gerosa M, Nicolato A, Foroni R, Zanotti B, Tomazzoli L, Miscusi M, et al: Gamma knife radiosurgery for brain metastases: a primary therapeutic option. J Neurosurg 97 (5 Suppl):515-524, 2002

8. Hawasli AH, Ray WZ, Murphy RK, Dacey RG Jr, Leuthardt EC: Magnetic resonance imaging-guided focused laser interstitial thermal therapy for subinsular metastatic adenocarcinoma: technical case report. Neurosurgery 70 (2 Suppl Operative):332-338, 2012

9. Lin NU, Lee EQ, Aoyama H, Barani IJ, Barboriak DP, Baumert BG, et al: Response assessment criteria for brain metastases: proposal from the RANO group. Lancet Oncol 16:e270-e278, 2015

10. Nath SK, Sheridan AD, Rauch PJ, Yu JB, Minja FJ, Vortmeyer AO, et al: Significance of histology in determining management of lesions regrowing after radiosurgery. J Neurooncol 117:303-310, 2014

11. Rahmathulla G, Recinos PF, Valerio JE, Chao S, Barnett GH: Laser interstitial thermal therapy for focal cerebral radiation necrosis: a case report and literature review. Stereotact Funct Neurosurg 90:192-200, 2012

12. Rao MS, Hargreaves EL, Khan AJ, Haffty BG, Danish SF: Magnetic resonance-guided laser ablation improves local control for postradiosurgery recurrence and/or radiation necrosis. Neurosurgery 74:658-667, 2014

13. Schwarzmaier HJ, Eickmeyer F, von Tempelhoff W, Fiedler
VU, Niehoff H, Ulrich SD, et al: MR-guided laser irradiation of recurrent glioblastomas. J Magn Reson Imaging 22:799803,2005

14. Sharma M, Balasubramanian S, Silva D, Barnett GH, Mohammadi AM: Laser interstitial thermal therapy in the management of brain metastasis and radiation necrosis after radiosurgery: an overview. Expert Rev Neurother 16:223232, 2016

15. Sloan AE, Ahluwalia MS, Valerio-Pascua J, Manjila S, Torchia MG, Jones SE, et al: Results of the NeuroBlate System first-in-humans Phase I clinical trial for recurrent glioblastoma: clinical article. J Neurosurg 118:1202-1219, 2013

16. Smith CJ, Myers CS, Chapple KM, Smith KA: Long-term follow-up of 25 cases of biopsy-proven radiation necrosis or post-radiation treatment effect treated with magnetic resonance-guided laser interstitial thermal therapy. Neurosurgery 79 (Suppl 1):S59-S72, 2016

17. Sneed PK, Mendez J, Vemer-van den Hoek JG, Seymour ZA, Ma L, Molinaro AM, et al: Adverse radiation effect after stereotactic radiosurgery for brain metastases: incidence, time course, and risk factors. J Neurosurg 123:373-386, 2015

18. Telera S, Fabi A, Pace A, Vidiri A, Anelli V, Carapella CM, et al: Radionecrosis induced by stereotactic radiosurgery of brain metastases: results of surgery and outcome of disease. $\mathbf{J}$ Neurooncol 113:313-325, 2013

19. Torcuator RG, Hulou MM, Chavakula V, Jolesz FA, Golby AJ: Intraoperative real-time MRI-guided stereotactic biopsy followed by laser thermal ablation for progressive brain metastases after radiosurgery. J Clin Neurosci 24:68-73, 2016

20. Torres-Reveron J, Tomasiewicz HC, Shetty A, Amankulor NM, Chiang VL: Stereotactic laser induced thermotherapy (LITT): a novel treatment for brain lesions regrowing after radiosurgery. J Neurooncol 113:495-503, 2013

\section{Disclosures}

Monteris Medical Corporation sponsored this study. Drs. Ahluwalia, Chiang, Dietrich, Laxton, Leuthardt, and Mohammadi are consultants for Monteris Medical Corporation. Dr. Barnett is a consultant for and owns stock in Monteris Medical Corporation.

\section{Author Contributions}

Conception and design: Chiang, Ahluwalia, Barnett, Tatter, Laxton, Mohammadi, Leuthardt, Chamoun, Judy, Asher, Essig, Dietrich. Acquisition of data: Chiang, Ahluwalia, Barnett, Tatter, Laxton, Mohammadi, Leuthardt, Chamoun, Judy, Asher, Essig, Dietrich. Analysis and interpretation of data: Chiang, Deng. Drafting the article: Chiang, Deng. Critically revising the article: Chiang, Ahluwalia, Barnett, Deng, Laxton, Mohammadi, Leuthardt, Chamoun, Judy, Asher. Reviewed submitted version of manuscript: Ahluwalia, Barnett, Deng, Tatter, Laxton, Mohammadi, Leuthardt, Chamoun, Judy, Asher, Essig, Dietrich. Approved the final version of the manuscript on behalf of all authors: Chiang. Study supervision: Chiang, Ahluwalia, Barnett, Tatter, Laxton, Mohammadi, Leuthardt, Chamoun, Judy, Asher, Essig, Dietrich.

\section{Supplemental Information}

\section{Previous Presentations}

A portion of this material was previously presented at the Society of NeuroOncology Annual Meeting in Scottsdale, Arizona, on November 18, 2016.

\section{Correspondence}

Veronica L. Chiang: Yale University, New Haven, CT. veronica. chiang@yale.edu. 